\title{
Towards minimum reporting standards for life scientists
}

\author{
The "minimum standards" working group ${ }^{1}$
}

\begin{abstract}
Transparency in reporting benefits scientific communication on many levels. While specific needs and expectations vary across fields, the effective use of research findings relies on the availability of core information about research materials, data, and analysis. In December 2017, a working group of journal editors and experts in reproducibility convened to create the "minimum standards" working group. This working group aims to devise a set of minimum expectations that journals could ask their authors to meet, and will draw from the collective experience of journals implementing a range of different approaches designed to enhance reporting and reproducibility (e.g. STAR Methods), existing life science checklists (e.g. the Nature Research reporting summary), and the results of recent meta-research studying the efficacy of such interventions (e.g. Macleod et al. 2017; Han et al. 2017).
\end{abstract}

The working group will provide three key deliverables:

- A "minimum standards" framework setting out minimum expectations across four core areas of materials (including data and code), design, analysis and reporting (MDAR)

- A "minimum standards" checklist intended to operationalize the framework by serving as an implementation tool to aid authors in complying with journal policies, and editors and reviewers in assessing reporting and compliance with policies

- An "elaboration" document or user guide providing context for the "minimum standards" framework and checklist

While all three outputs are intended to provide tools to help journals, researchers and other stakeholders with adoption of the minimum standards framework, we do not intend to be prescriptive about the precise mechanism of implementation and we anticipate that in many cases they will be used as a yardstick within the context of an existing reporting system. Nevertheless, we hope these tools will provide a consolidated view to help raise reporting standards across the life sciences.

The working group will pilot these deliverables with a small number of journals and ask them to use the checklist within the editorial review and author revision process. Authors and editors will be asked to qualitatively evaluate the utility of the checklist. We will also seek out qualitative feedback on the framework, checklist, and elaboration document from a small stakeholder group of researchers and experts in reproducibility and transparent reporting. Feedback from this process will be used to consider next steps, which might include revisions to the content of the checklist. Following this process, journals will be free to use the checklist in ways that support their workflows.

The data and materials for this evaluation will be made available as the supplemental materials to this preprint after the initial pilot period, which is expected to end in September, 2019. Data will only be provided in aggregate form, that is, not parsed by individual article or journal, so as to respect the confidentiality of responses.

An advantage of aligning on minimum standards is consistency in policies and expectations across journals, which is beneficial for authors as they prepare papers for publication and for reviewers as they assess them. We also hope that other major stakeholders engaged in the research cycle, including institutional review bodies and funders, will see the value of agreeing on this type of reporting standard as a minimum expectation, as broad-based endorsement from an early stage in the research life cycle would provide important support for overall adoption and implementation.

\footnotetext{
${ }^{1}$ Karen Chambers (Wiley; kchamber@wiley.com), Andy Collings (eLife; a.collings@elifesciences.org), Chris Graf (Wiley; cgraf@wiley.com), Veronique Kiermer (Public Library of Science; vkiermer@plos.org; first corresponding author), David Mellor (Center for Open Science; david@cos.io), Malcolm Macleod (University of Edinburgh; Malcolm.macleod@ed.ac.uk), Sowmya Swaminathan (Nature Research/Springer Nature; s.swaminathan@us.nature.com, second corresponding author), Deborah Sweet (Cell Press/Elsevier; dsweet@cell.com), Valda Vinson (Science/AAAS; vvinson@aaas.org)
} 